\title{
Chronological Variations of Children Poisoning Causes in Zahedan, South of Iran
}

\author{
Simin Sadeghi-Bojd ${ }^{1}$; Ali Khajeh ${ }^{2, *}$ \\ ${ }^{1}$ Department of Pediatrics, School of Medicine, Zahedan University of Medical Sciences, Zahedan, IR Iran \\ ${ }^{2}$ Children and Adolescents Health Research Center, Zahedan University of Medical Sciences, Zahedan, IR Iran \\ ${ }^{*}$ Corresponding author: Ali Khajeh, Children and Adolescents Health Research Center, Zahedan University of Medical Sciences, Zahedan, IR Iran. Tel: +98-5412421638, E-mail: drkha- \\ jehneuro@gmail.com
}

Received: April 18, 2014; Revised: May 1, 2014; Accepted: May 11, 2014

\begin{abstract}
Background: Poisoning is a common pediatric emergency among children and adolescents in the Emergency Department of Zahedan University of Medical Sciences hospital.

Objectives: The aim of this study was comparing the characteristics and variations of pediatric poisoning between two retrospective studies (1998 and 2008). We hypothesized that the epidemiology of pediatric patients admitted for poisoning is related to variations of environmental agents and drug usage.

Patients and Methods: Records of 170 patients from 1998 and 147 from 2008 with acute poisoning were retrospectively evaluated and compared.

Results: Poisoning mostly occurred in children younger than fiveyears old via oral route(72.94\%-87\%)and by single exposure(94.12\%-96.6\%). It was also noted that $86.8 \%-90 \%$ of cases were accidentally poisoned. Drugs were the most common poisoning agents in both studies (52.94\% and 37.41\%, respectively) and analgesics-antipyretics were the most common poisoning drugs. Drug poisoning was more common among children under five years old in both the studies. Neurological signs including lethargy and coma were the main presenting signs. About $80 \%-95 \%$ of cases were referred to the hospital within three hours of poisoning and supportive-symptomatic therapy was provided to them; charcoal/naloxone was administered for most of the patients (26.2\% in 2008 and $21 \%$ in 1998 ). Mortality rate due to drug poisoning was 3-4 cases in both studies; but, non-drug poisoning mortality rate was higher.

Conclusions: Preventable accidental poisoning is a significant cause of morbidity in children in developing countries. The study provided information on evolving trends and the need for increasing awareness about potential toxins as well as appropriate storage of toxins in the house to reduce the occurrence of accidental poisoning.
\end{abstract}

Keywords: Poisoning; Emergency Medical Services; Chronology

\section{Background}

Poisoning is one of the most common medical emergencies in young children and accounts for a significant proportion of emergency department visits in adolescents (1). Incidence of childhood poisoning reported in various studies ranges from $0.33 \%$ to $7.6 \%$. Cause of poisoning varies with age, gender, education, and cultural background and it also shows seasonal variations (2). Poisoning may be unintentional or intentional. Unintentional poisonings comprise $80 \%-85 \%$ of all reported cases, whereas intentional poisonings comprise the other $10 \%$ $15 \%$ (1). In a report by the American Association of Poison Control Centers (AAPCC), more than $50 \%$ of poisonings occur in children under six years old. Almost all of these exposures are unintentional and this reflects the propensity of young children to put virtually anything in their mouth (3). Children who are more at risk are between one to four years old, male, rather hyperactive, and have increased finger-mouth activity and/or pica.

Poisoning in children 6-12 years old is lesser common, involving only $6 \%$ of all reported pediatric exposures. A second peak in pediatric exposures occurs in adolescence; it is primarily intentional and often results in more severe toxicity (3).

The most important difference between pediatric and adult poisonings is types of agents. In adults, higher percentages of poisoning cases are due to psychopharmacologic drugs (sedatives, tranquilizers and antidepressants), whereas in children, there is a much higher frequency of exposure to household items and personal care products and plants (1). More than 90\% of toxic exposures in children occur inside the house and they mostly involve a single substance. Ingestion accounts for a vast majority of exposures, and a minority happens via dermal, inhalational, and ophthalmic routes.

Approximately 50\% of cases involve nondrug substances such as cosmetics, personal care items, cleaning solutions, plants, and foreign bodies. Pharmaceutical preparations account for the remainder of exposures, among

Copyright @ 2014, Zahedan University of Medical Sciences; Published by Kowsar Corp. This is an open-access article distributed under the terms of the Creative Commons Attribution License, which permits unrestricted use, distribution, and reproduction in any medium, provided the original work is properly cited. 
which, analgesics, topical preparations, cough and cold products and vitamins are the most commonly reported categories (2).

The majority of poisoning exposures in children under six years old can be managed without direct medical interventions, either because the involved product is not toxic or the quantity of the involved material is not sufficient to produce clinically relevant toxic effects. However, a number of substances are potentially highly toxic to toddlers even in small doses (3). In the recent years, carbon monoxide, prescription opioids, antidepressants and cardiovascular drugs have been the leading causes of poison-related fatalities in young children. Mortality rate due to poisoning is 3\%-5\% (2). Although children comprise the majority of exposures in the population, only $2 \%$ of the reported deaths occur in this age group (3).

In this study, we attempted to share the experience of childhood poisoning of a single center over two periods: 1998 and 2008. Demographic and clinical characteristics as well as changes over time with regard to diagnosis and treatment were presented.

\section{Objectives}

The main purpose of the study was to compare the characteristics and variations in pediatric poisoning and determine the differences of cause, age, gender and outcome of poisoning between two retrospective studies (1998 and 2008) to help in diagnosis, management and prevention of poisoning. We hypothesized that the epidemiology of pediatric patients admitted for poisoning was related to variation in environmental agents and drug usage.

\section{Patients and Methods}

In this retrospective study, the records of children aged 0-15 years old admitted to the pediatric emergency ward in Ali Asghar Hospital in Zahedan, Iran, for acute poisoning in 1998 were reviewed and compared with a report by Khajeh et al. which evaluated the epidemiological parameters of childhood poisoning cases admitted to Ali Ebne Abitaleb Hospital in Zahedan in 2008 (4).

The inclusion criteria were patients less than 15 years old admitted to the two mentioned hospitals with main diagnosis of acute poisoning based on history and practitioner's judgment. The occurrence of childhood poisoning was described according to time trends, age and gender of the child, route of exposure, symptoms at presentation to the emergency room, role of the child or others in poisoning, substances involved in poisoning, the duration between exposure and admission, hospitalization duration, and outcome. Categorical variables were expressed as percentages and continuous variables as mean and SD. The study protocol was approved by the Ethics Committee of Zahedan University of Medical Sciences.

\begin{tabular}{lcc}
\hline \multicolumn{2}{c}{ Table 1. Gender and Age Distributions of Poisoned Cases ${ }^{\mathrm{a}}$} \\
\hline \multicolumn{1}{c}{$\mathbf{1 9 9 8}$} & $\mathbf{2 0 0 8}$ \\
\hline Age, $\mathbf{y}$ & & \\
$<5$ & $124(72.94)$ & $128(87)$ \\
$>5$ & $46(27)$ & $19(23)$ \\
Gender & & \\
Male & $81(47)$ & $83(56.4)$ \\
Female & $89(53)$ & $64(43.6)$ \\
\hline
\end{tabular}

a Data are presented as No. (\%).

\section{Results}

In 1998 and 2008, 370 and 177 children were admitted to the pediatric emergency room due to accidental poisoning, respectively. Complete clinical data were obtained and compared between the two time periods.

\subsection{Age and Sex}

The patients consisted of 81 (47\%) boys and 89 (53\%) girls in 1998 and 83 (56.4\%) boys and 64 (53.4\%) girls in 2008, indicating the increase of poisoned boys in the recent years. There were $124(72.94 \%)$ children under five years old and 46 (27\%) were over 5 years old (1998). In the 2008 study, 128 (87\%) cases were under 5 years old (Table 1 ).

\subsection{Route of Poisoning}

The most common route of poisoning was ingestion of poison (94.12\%-96.6\%) in both studies. The remaining patients were poisoned by the respiratory route (3.4\%) such as CO gas and hashish in 2008; in addition, there were other routes such as inhalation and topical exposure in 1998. The most common season for poisoning was autumn (40.1\%), followed by winter (23.1\%), summer (19.7\%), and spring (17\%) in 2008, but it occurred most commonly in winter in 1998, which was a difference between the two studied years.

\subsection{Agents Involved}

Drugs were the most common agents causing poisoning in both studies (52.94\% and $37.41 \%$ respectively), followed by ingestion of agricultural pesticides/insecticides $(18.7 \%, \mathrm{n}=32)$, kerosene $(9.4 \%, \mathrm{n}=16)$, opioids ( $7 \%$, $\mathrm{n}=12)$, and corrosive agents $(2.9 \%, \mathrm{n}=5)$ in 1998. In 2008, the second cause was kerosene $(23.12 \%, \mathrm{n}=34)$, followed by hashish $(17.68 \%, \mathrm{n}=26)$, agricultural pesticides/insecticides $(6.85, \mathrm{n}=10)$, unknown causes $(5.44 \%, \mathrm{n}=8)$, and corrosives ( $4.79 \%, \mathrm{n}=7$ ). Benzodiazepines were the most common causes of medication-related poisonings in both studies, which comprised $45.5 \%$ and $44.5 \%$ of cases, respectively. Tricyclic antidepressants were the second common drug in both groups $(24.44 \%, \mathrm{n}=22 ; 35.5 \%, \mathrm{n}=$ 20 , respectively) (Table 2 ). Palliative medication and phe 


\begin{tabular}{lcc}
\hline \multicolumn{3}{l}{ Table 2. Chronologic Variations in Cause of Poisoning } \\
\hline & $\mathbf{1 9 9 8}$ & $\mathbf{2 0 0 8}$ \\
\hline Kerosene & $16(9.4)$ & $34(23.1)$ \\
\hline Corrosive Agent & $5(2.9)$ & $7(4.8)$ \\
\hline Drug & $90(52.94)$ & $55(37.4)$ \\
\hline Benzodiazepines & $41(45.55)$ & $25(44.5)$ \\
\hline Tricyclic antidepressant & $22(24.44)$ & $20(35.5)$ \\
\hline Palliative medication & $6(6.66)$ & $5(10)$ \\
\hline Phenothiazines & $12(13.33)$ & 0 \\
\hline Unknown medication & $9(10)$ & $5(10)$ \\
\hline Agricultural pesticides/insecticides & $32(18.7)$ & $10(6.8)$ \\
\hline Plants & $5(2.9)$ & - \\
\hline Opium & $12(7)$ & $26(17.7)$ \\
\hline Unknown & $10(5.88)$ & $8(5.4)$ \\
\hline Others & - & $7(4.8)$ \\
\hline
\end{tabular}

$\mathrm{a}$ Data are presented as No.(\%).

\begin{tabular}{lcc}
\hline \multicolumn{3}{l}{ Table 3. Distribution of Poisoning Agents According to Age ${ }^{\mathrm{a}}$} \\
\hline Cause, $\mathbf{y}$ & $\mathbf{1 9 9 8}$ & $\mathbf{2 0 0 8}$ \\
\hline Drug & & \\
$<5$ & $65(52.41)$ & $46(35.9)$ \\
$>5$ & $25(52.34)$ & $9(47.3)$ \\
\hline Kerosene & & \\
$<5$ & $14(11.29)$ & $30(23.4)$ \\
$>5$ & $2(4.34)$ & $4(21)$ \\
\hline Opium & & \\
$<5$ & $11(8.87)$ & $25(19.53)$ \\
$>5$ & $1(2.17)$ & $1(5.26)$ \\
\hline Others & & $27(21)$ \\
$<5$ & $34(27.41)$ & $5(26.3)$ \\
\hline$>5$ & $18(39.13)$ & \\
\hline Total & & $128(100)$ \\
\hline$<5$ & $124(100)$ & $19(100)$ \\
\hline$>5$ & $46(100)$ & \\
\hline a Data are presented as No. (\%). &
\end{tabular}

\begin{tabular}{lcc}
\hline \multicolumn{2}{l}{ Table 4. Distribution of Patients Outcomes Over Time ${ }^{\mathrm{a}, \mathrm{b}}$} \\
\hline \multicolumn{1}{c}{$\mathbf{1 9 9 8}$} & $\mathbf{2 0 0 8}$ \\
\hline Admission & & \\
Emergency room & $76(44.70)$ & $147(100)$ \\
Pediatric ward & $32(18.82)$ & $17(11.6)$ \\
PICU & $4(2.35)$ & $3(2)$ \\
Mortality rate & $4(2.35)$ & $3(2)$ \\
\hline
\end{tabular}

\footnotetext{
${ }^{a}$ Abbreviation: PICU, pediatric intensive care unit.

${ }^{b}$ Data are presented as No. (\%).
}

nothiazines were other common medications. Opioids were more common in $2008(17.68 \%, \mathrm{n}=26)$ probably due to increasing opioid usage in the recent years. Poisoning caused by medications was more common in children under five years old in both studies (Table 3).

Of all the cases referred to the emergency unit in 1998, $80 \%$ arrived within the first two hours and $95 \%$ in the first six hours following poisoning; however, in 2008, 96.3\% were referred after three hours and $4.6 \%$ over one to three days of poisoning. Respiratory distress and unconsciousness were the most common complaints at presentation to the hospital in both studies. In the 2008 study, $11.6 \%$ patients were admitted to the pediatric ward, of which three (3\%) were admitted to the pediatric intensive care unit (ICU)due to severe complications (respiratory distress); in $1998,18.8 \%$ of the patients were admitted to the pediatric ward and $3 \%$ of them were admitted to the pediatric ICU.

\subsection{Treatment and Duration of Hospital Stay}

In most of the cases in both studies, the provided treatment was nonspecific and included general decontamination and supportive-symptomatic therapy. In total, $55.6 \%$ of patients did not need any intervention such as gastric lavage or antidote administration. In $26.2 \%$ of cases, charcoal or naloxone was administered and $17.2 \%$ underwent gastric lavage. In both studies, mortality due to poisoning was similar (2.35\% and $2 \%$, respectively).Among the occurred deaths, two were due to drug poisoning and two due to hydrocarbon intoxication in 1998; however, all deaths were due to kerosene ingestion in children less than five years old in 2008. The cause of death was dysrhythmia in two children and the other patients were discharged with full recovery (Table 4).

\section{Discussion}

Acute poisoning is an important cause of emergency unit admissions. Identification and documentation of epidemiological aspects and other variables in childhood poisoning are of great importance for treatment planning and determination of proper preventive measures. The results of this study reflected the epidemiology of childhood poisonings and temporal variations over time. The most important findings of this study were the increasing cases of opioid poisoning and decreasing cases of pesticides poisoning in the recent years.

In this study, the male/female ratio of patients was about 1 (0.99 to 1.05) in both studies, consistent with previous reports $(2,5)$; however, in other reports, poisoning was more common among boys $(6,7)$. This was probably because boys are more active and curious than girls. Ozdemir et al. described two distinct peaks of age, boys between 1-5 and girls between 13-16 years old, in which poisoning was more common (8). The most prevalent (72.94\%-87\%) age group was children under five years of age in both studies, which was similar to the results of previous studies $(6,7,9,10)$. Andiran and Sarikayalar 
found that in 489 poisoning cases, poisonings among children less than 10 years old was more frequent in boys, whereas poisonings in children more than 10 years of age was more commonly observed in females (6).

Ozdemir et al. observed that $61.2 \%$ of cases were younger than six years old (8). In this age group, putting small foreign objects like drugs in mouth was a cause of poisoning. As reported in the literature, the most common route of poisoning was ingestion $(96.6 \%)(2,6)$. In this study, $37.41 \%-52.94 \%$ of all poisonings were due to drugs. This may be due to their easy accessibility. These findings were similar to previous reports $(48.3 \%-64 \%)(6,8)$. In this study, benzodiazepines and tricyclic antidepressants were the two groups of drugs causing almost all single drug-related poisonings in both time periods; this was followed by opioid drugs in 2008.

Many studies listed analgesics and benzodiazepines as the most common drugs in childhood poisoning $(7,8,11)$. Azemi et al. reported that among drugs, the most dominant causes were benzodiazepines (10 cases) and metoclopramide (4 cases) and among pesticides, it was malathion, which was also seen in 1998 (7). Hashish was a cause of poisoning in $17.68 \%$ of patients in 2008 , which was different from 1998. One of the reasons for the high incidence of poisoning by opioids was due to neighboring countries, Afghanistan and Pakistan, from which these drugs are easily available. Methadone poisoning is increasing in this society due to higher doses of methadone in medication syrups (12). In similar reports from Turkey and Pakistan, kerosene was one of the most common causes of acute pediatric poisoning $(10,13,14)$. This may reflect socioeconomic reasons as well as the use of hydrocarbons as a fuel by people in developing countries. Although gastrointestinal symptoms and psychological signs have been reported as the most frequent complaints of pediatric poisonings (8) in other studies, in these two studies, neurological signs including lethargy and unconsciousness were the main presenting complaints (5). Nausea and vomiting were the most common complaints at presentation to the hospital (42.3\%), followed by unconsciousness (2). The majority of cases were exposed to accidental poisoning (86.8\%-90\%) in both periods; this occurred mostly in children under five years old (93\%); but in Turkey, accidental usage of drugs constituted the majority (73\%) of all poisonings and only $25.6 \%$ occurred as a suicidal attempt (2). In another study, the suicidal poisoning rate was $23.8 \%$ among all poisoned patients, $98.9 \%$ of whom were adolescents $(9,15)$. Veale reported that intentional poisoning was frequent in adults, of which $64.3 \%$ were females (11). Pawlowicz et al. also reported that poisoning was intentional in $75 \%$ of cases and was often due to intoxication by alcohol (16). In both studies, $80 \%-95 \%$ of cases were referred to hospital within three hours, which was higher than that reported in similar studies (5). Sahin et al. reported that $95.7 \%$ of patients presented within six hours following poisoning (2).

Supportive-symptomatic therapy was provided to most patients; charcoal/naloxone was administered in
$26.2 \%$ of patients in 2008 and $21 \%$ in 1998 . In the two prospective studies, gastric lavage was used in $17.2 \%-20 \%$ of patients with acute poisoning. Gastric lavage was performed in $41.2 \%$ of patients in another study (2). Mortality due to acute poisoning in our two series was 3-4 cases. In a previous study, a mortality rate of $1.9 \%$ was reported among patients admitted with acute poisoning (8). This decrease in mortality may be attributed to greater parental awareness and technological advances in the ICU. Many studies on childhood poisoning have reported higher mortality rates for suicidal poisoning compared with accidental poisoning.

Our research had some limitations. It was performed retrospectively and all the medical data might not be recorded in the patients' files in the emergency ward.

Most poisoning cases reported in children, especially in infants and children younger than five years old, are due to accidental ingestions. Preventable accidental poisoning is still a significant cause of morbidity among children in developing countries. Drugs, corrosive agents and kerosene agents are the most frequent agents responsible for poisoning in the pediatric population.

\section{Acknowledgements}

The authors wish to thank all the personnel of Pediatric Emergency, Children Ward, and the Archive of Ali-ebneAbitalib Hospital of Zahedan.

\section{Authors' Contributions}

Study concept, design analysis and interpretation of data: Ali Khajeh; drafting of the manuscript: Simin Sadeghi.

\section{References}

1. Kumar MR, Kumar GP, Babu PR, Kumar SS, Subrahmanyam BV, Veeraprasad $\mathrm{M}$, et al. A retrospective analysis of acute organophosphorus poisoning cases admitted to the tertiary care teaching hospital in South India. Ann Afr Med. 2014;13(2):71-5.

2. Sahin S, Carman KB, Dinleyici EC. Acute poisoning in children; data of a pediatric emergency unit. Iran J Pediatr. 2011;21(4):47984.

3. Blake D, Dalton S, Gunja N. Transporting children with toxicological emergencies. Emerg Med Australas. 2014;26(3):279-85.

4. Khajeh A, Kaykha M, Hanafi-Bojd H. Patterns of acute poisoning in childhood and relative factors in Zahedan, Southeast Iran. Shiraz E-Med J. 2012;13(3).

5. Gheshlaghi F, Piri-Ardakani MR, Yaraghi M, Shafiei F, Behjati M. Acute poisoning in children; a population study in isfahan, iran, 2008-2010. Iran J Pediatr. 2013;23(2):189-93.

6. Andiran N, Sarikayalar F. Pattern of acute poisonings in childhood in Ankara: what has changed in twenty years? Turk J Pediatr. 2004;46(2):147-52.

7. Azemi M, Berisha M, Kolgeci S, Bejiqi R. Frequency, etiology and several sociodemographic characteristics of acute poisoning in children treated in the intensive care unit. Mater Sociomed. 2012;24(2):76-80.

8. Ozdemir R, Bayrakci B, Teksam O, Yalcin B, Kale G. Thirtythree-year experience on childhood poisoning. Turk J Pediatr. 2012;54(3):251-9.

9. Mutlu M, Cansu A, Karakas T, Kalyoncu M, Erduran E. Pattern of pediatric poisoning in the east Karadeniz region between 2002 and 2006: increased suicide poisoning. Hum Exp Toxicol. 2010;29(2):131-6. 
10. Abbas SK, Tikmani SS, Siddiqui NT. Accidental poisoning in children.J Pak Med Assoc. 2012;62(4):331-4.

11. Veale DJ, Wium CA, Muller GJ. Toxicovigilance. I:A survey of acute poisonings in South Africa based on Tygerberg Poison Information Centre data. S Afr Med J. 2013;103(5):293-7.

12. Shadnia S, Rahimi M, Hassanian-Moghaddam H, Soltaninejad K, Noroozi A. Response to letter: "Methadone toxicity: comparing tablet and syrup formulations during a decade in an academic poison center of Iran". Clin Toxicol (Phila). 2014;52(2):154.

13. Chhetri UD, Ansari I, Shrestha S. Pattern of pediatric poisoning and accident in Patan Hospital. Kathmandu Univ Med J (KUMJ).
2012;10(39):39-43.

14. Gonullu H, Karadas S, Oncu MR, Dulger AC, Keskin S. The analysis of the cases of aspired fuel oil and gasoline through siphonage method. J Pak Med Assoc. 2013;63(3):383-4.

15. Azkunaga B, Mintegi S, Salmon N, Acedo Y, Del Arco L. [Poisoning in children under age 7 in Spain. Areas of improvement in the prevention and treatment]. An Pediatr (Barc). 2013;78(6):355-60.

16. Pawlowicz U, Wasilewska A, Olanski W, Stefanowicz M. Epidemiological study of acute poisoning in children: a 5-year retrospective study in the Paediatric University Hospital in Bialystok, Poland. Emerg Med J. 2013;30(9):712-6. 